\title{
Simulation de l'énergie solaire directe au pas de temps de la minute. L'exemple de Saint-Chamas (Marseille)
}

\author{
Mlle M. J. Mejon $\left({ }^{*}\right)$, Ph. Bois $\left({ }^{*}\right)$ et R. Lestienne $(* *)$ \\ Institut de Mécanique (I.M.G.), Domaine Universitaire, BP 53, 38041 Grenoble Cedex, France \\ (Reçu le 27 juin 1979, révisé le 11 octobre 1979, accepté le 15 octobre 1979)
}

Résumé. - Les données enregistrées pendant le mois de juillet 1977 à Saint-Chamas au pas de temps de la minute sont comparées aux résultats de simulation sur ordinateur obtenus à partir des trois schémas suivants :

1) Modèle à chaîne de Markov à deux états journaliers et à tout ou rien pour l'éclairement direct (soleil/passages nuageux);

2) Modèle à chaîne de Markov à trois états journaliers et à tout ou rien pour l'éclairement ;

3) Modèle à chaîne de Markov à deux états journaliers et à éclairement simulé d'après les histogrammes empiriques.

Ce dernier schéma est nettement plus satisfaisant que les deux premiers et pourrait convenir pour les applications pratiques de prédiction du comportement de systèmes solaires à faible inertie.

\begin{abstract}
The data on direct solar radiation recorded at the Saint-Chamas station during the month of July 1977 with a one minute binning are compared to the outputs of a computer simulation obtained within the three following schemes :

1) A two-states Markov chain for daily weather and a yes-no output for the direct radiation (according to sunshinning or cloudiness);

2) A three-states Markov chain for daily weather and a yes-no output for the direct radiation ;

3) A two-states Markov chain for daily weather and a direct radiation output that is sorted according to the empirical histograms.
\end{abstract}

The last scheme is clearly more satisfactory than the two others and looks convenient for practical applications to forecasting the behaviour of low inertia solar systems.

1. Introduction. - 1.1 BUt DE LA SimUlation. Certaines installations solaires utilisent l'énergie solaire directe ; c'est le cas notamment des centrales électrosolaires à concentration de type THEMIS [7] ou THEK. Cette énergie reçue est très variable d'un jour à l'autre, mais aussi très fluctuante au long de la journée, du fait de l'existence de passages nuageux intermittents. Il est donc important de connaître la structure statistique fine de l'énergie reçue à un pas de temps suffisamment petit pour décrire le comporte-

$\left({ }^{*}\right)$ Institut de Mécanique de Grenoble.

(**) PIR DES-C.N.R.S., Paris (Programme Interdisciplinaire de Recherches et de développement de l'Energie Solaire). ment des systèmes à faible inertie. Malheureusement, on dispose actuellement de trop peu de données historiques des mesures de rayonnement direct à un pas de temps fin, la minute par exemple. Il est donc exclu d'utiliser ces données historiques brutes pour le dimensionnement ou la définition du mode de gestion d'une installation solaire.

Nous présentons donc des procédés de simulation de données d'énergie directe à pas de temps fin, calés sur la petite série de données observées. Comme ces séries sont encore très courtes, le calage est assez grossier; aussi cet article est-il surtout méthodologique. Il est clair qu'une série de données simulées ne contient pas plus d'information statistique que la série historique qui a servi à définir la simulation. Mais les séries simulées sont d'une part souvent plus 
faciles à utiliser que des séries historiques (volume de données à lire beaucoup plus réduit) et d'autre part, du fait de leur longueur, elles peuvent décrire un plus grand nombre de situations que celles observées sur les données.

1.2 Principe GéNéral De la Simulation De DONNÉES D'ÉNERGIE DIRECTE. - La simulation doit respecter le plus fidèlement possible le phénomène physique. Dans notre cas, il faudra, a priori :

- Respecter les lois de probabilité marginales des énergies quotidiennes : c'est-à-dire simuler, en espérance mathématique, autant de jours de beau temps ou de mauvais temps que ceux observés.

- Respecter, en terme de probabilité, les lois de probabilité conditionnelles des énergies quotidiennes compte tenu du temps de la veille.

- Respecter au sein des journées simulées, les lois de probabilité des durées des éclaircies et des passages nuageux et ceci selon le type de temps journalier.

- Respecter les lois de répartition des valeurs du rayonnement au sein des éclaircies et des passages nuageux en tenant compte du moment de la journée et du type de temps.

Nous présentons trois schémas de simulation en définissant leurs caractéristiques et en comparant leurs performances.

2. Données ayant servi au calage. - Depuis 1977 EDF enregistre, à Saint-Chamas, près de Marseille [7], l'énergie solaire directe normale au soleil au pas de temps de la minute. Ces données ont été vérifiées :

- d'une part, en contrôlant que le nombre d'heures de soleil calculé à partir de ces mesures (énergie reçue supérieure à $100 \mathrm{~W} / \mathrm{m}^{2}$ ) est voisin de l'insolation mesurée à Marseille-Marignane, station météorologique assez proche,

- d'autre part, en comparant les énergies mesurées aux énergies maximales possibles en fonction de la date de l'année et de l'heure, pour ce site.

Afin de travailler sur des échantillons homogènes du point de vue météorologique, nous avons regroupé les valeurs mois par mois; dans cet article, le mois ayant servi au calage est le mois de juillet 1977. Il aurait été intéressant de pouvoir disposer d'une série de plusieurs mois de juillet pour caler les schémas de simulation; par contre, il aurait été certainement imprudent de travailler sur des échantillons rassemblant plusieurs mois successifs, les variations saisonnières étant importantes dans cette région (cf. J. Goussebaile [2] ou R. Lestienne [3]).

Dans la suite, nous appellerons éclairement direct, la puissance solaire directe reçue sur un plan perpendiculaire au soleil.

Nous avons également utilisé, pour la simulation des séquences de jours, les longues séries d'insolation journalière disponibles à Marseille-Marignane (27 ans disponibles).
3. Premier schéma de simulation : modèle à deux états journaliers et à tout ou rien pour l'éclairement. Ce premier schéma est le plus simple : il part d'un classement des journées en deux types : mauvais temps (état appelé état 0 ) et beau temps (état 1) et considère que lorsqu'il y a une éclaircie, l'éclairement atteint la valeur maximale observée au sol, fonction de la date et de l'instant considéré, alors que lorsqu'il y a un passage nuageux, cet éclairement est nul.

3.1 Simulation Des Jours Successifs. - Avant de simuler les éclairements au sein de chaque jour, nous avons commencé par simuler des chroniques de journées successives. Pour cela, compte tenu du fait qu'il y a une bonne liaison entre l'énergie journalière et l'insolation journalière (cf. [5] et [6]), nous avons étudié les longues séries d'insolation journalière à Marseille-Marignane.

Il y a dépendance d'un jour à l'autre; les journées ont tendance à se grouper par séquences de beau et de mauvais temps. Il nous a donc semblé nécessaire de distinguer au minimum deux types de journées :

- celles que nous appellerons mauvais temps (état 0 ), lorsque le rapport d'insolation $\mathrm{SS}(\mathrm{J}) / \mathrm{SS}_{0}(\mathrm{~J})$ est inférieur à 0,$5 ; \mathrm{SS}(\mathrm{J})$ étant l'insolation mesurée le jour $\mathrm{J}$ et $\mathrm{SS}_{0}(\mathrm{~J})$ l'insolation potentielle, durée astronomique du jour $\mathrm{J}$, fonction de la date et de la latitude,

- beau temps (état 1) : rapport d'insolation supérieur à 0,5 .

3.1.1 Analyse en chaine de Markov des jours successifs. - A partir d'un échantillon rassemblant les observations journalières de la duré de l'insolation pour un mois donné (par exemple juillet) sur plusieurs années (par exemple de 1949 à 1975), on détermine :

- les probabilités marginales $P_{0}$ et $1-P_{0}=P_{1}$ de mauvais temps et beau temps,

- les probabilités conditionnelles :

$P_{00}$ : probabilité qu'il fasse mauvais le jour $\mathrm{J}+1$ sachant qu'il a fait mauvais le jour $\mathbf{J}$.

$P_{01}=1-P_{00}$ : probabilité qu'il fasse beau le jour $\mathbf{J}+1$ sachant qu'il a fait mauvais le jour $\mathbf{J}$.

$P_{10}$ : probabilité qu'il fasse mauvais le jour $\mathrm{J}+1$ sachant qu'il a fait beau le jour $J$.

$P_{11}=1-P_{10}$ : probabilité qu'il fasse beau le jour $\mathbf{J}+1$ sachant qu'il a fait beau le jour $\mathbf{J}$.

On notera que, dans le cas d'une chaîne de Markov d'ordre 1, seule deux des six valeurs des probabilités précédentes sont indépendantes [4]. Les valeurs ont été calculées par J. Goussebaile [2] et R. Lestienne [3] sur 21 stations du Sud-Est de la France. Deux résultats importants apparaissent :

- Pour une station donnée, évolution régulière des probabilités marginales et conditionnelles le long des douze mois de l'année (cf. Fig. 1);

- Pour un mois donné, mais pour l'ensemble des stations, évolution spatiale régulière (cf. Fig. 2). 


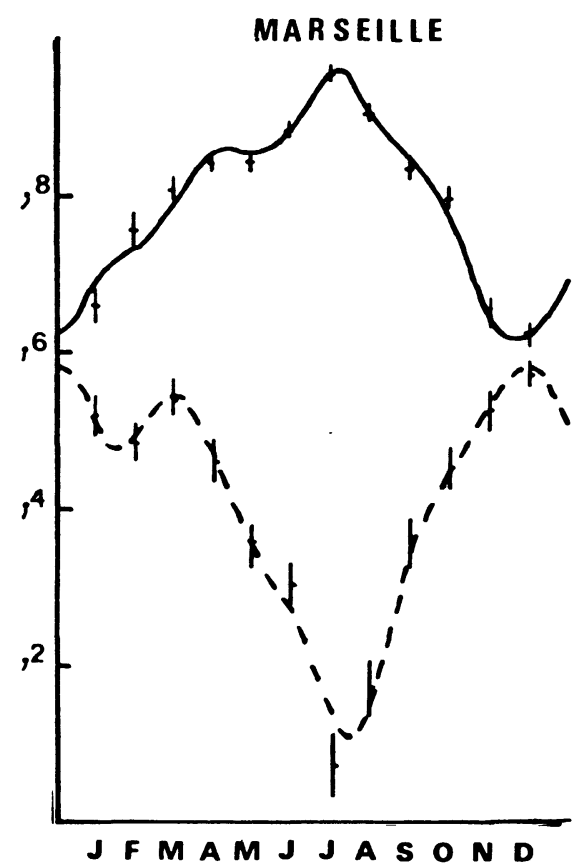

Fig. 1. - Variation saisonnière des probabilités beau temps-beau temps $P_{11}$ (traits pleins) et mauvais temps-mauvais temps $P_{00}$ (tirets). Les bâtonnets donnent une idée de l'incertitude.

[Seasonnal variation of the transition probabilities $P_{11}$ (fine weather-fine weather, continuous line) and $P_{00}$ (bad weather-bad weather, broken line). Vertical dashes give measurement uncertainties.]

Le modèle en chaîne de Markov d'ordre 1, est donc robuste tant du point de vue saisonnier que du point de vue spatial.

En juillet, on trouve pour Marseille-Marignane :

$P_{0}=0,04$ Probabilité marginale de mauvais temps.

$P_{1}=0,96$ Probabilité marginale de beau temps.

$P_{00}=0,12$ Probabilité de mauvais temps si la veille il y avait mauvais temps. D'où $P_{01}=1-P_{00}=0,88$.

$P_{11}=0,96$ Probabilité de beau temps si la veille il y avait beau temps. D'où

$$
P_{10}=1-P_{11}=0,04 \text {. }
$$

3.1.2 Simulation. - Pour simuler une chronique de jours, le procédé est alors très simple :

- Premier jour : on tire au hasard une variable $Z$ répartie uniformément sur $0-1$

si $Z \leqslant P_{0}$ le premier jour est un jour de mauvais temps;

si $Z>P_{0}$ le premier jour est un jour de beau temps.

- Deuxième jour et jours suivants. On tire une valeur de $Z$ :

- Si la veille du jour à simuler était de mauvais temps :

si $Z \leqslant P_{00}$ le mauvais temps se poursuit, $\mathrm{J}+1$ est mauvais : si $Z>P_{00}$ le mauvais temps s'arrête, le jour $\mathrm{J}+1$ est beau.

- Si la veille était un jour de beau temps :

si $Z \leqslant P_{10} \quad \mathrm{~J}+1$ sera de mauvais temps ; et ainsi de suite (cf. Lestienne [4]).

3.2 Simulation des Durées Des SÉquences D'ÉCLAIRCIES ET DE PASSAGES NUAGEUX AU SEIN D'UNE JOURNÉE SELON SON TYPE DE TEMPS. - 3.2.1 Séparation entre éclaircie et passage nuageux. - Pour des raisons de simplicité et compte tenu des seuils de démarrage de certains capteurs solaires, on a considéré que l'on était au sein d'une éclaircie tant que l'éclairement dépassait $250 \mathrm{~W} / \mathrm{m}^{2}$; les résultats sont d'ailleurs voisins si l'on prend des seuils de 200 ou $300 \mathrm{~W} / \mathrm{m}^{2}$. On a alors déterminé, pour chacun des deux types de temps :

- $F_{\mathrm{ON}}(d)$, fonction de répartition empirique des durées des séquences de passages nuageux pour les journées du type mauvais temps. De même $F_{1 \mathrm{~N}}(d)$ pour les journées du type beau temps.

- $F_{\mathrm{OE}}(d)$, fonction de répartition empirique des durées des séquences d'éclaircies pour le type mauvais temps; de même $F_{1 \mathrm{E}}(d)$ pour le type beau temps.

Les figures 3 et 4 présentent ces quatre fonctions. On constate leurs différences; il resterait évidemment à les déterminer sur des échantillons plus vastes.

\subsubsection{Simulation des séquences éclaircies-passages} nuageux au sein d'une journée J. - On connaît (cf. 3.1), le type de temps de la journée $J$. On génère alors un nombre $Z$ aléatoire uniforme sur $0-1$. La durée de la première séquence $d_{1}$ est définie par :

$F_{\text {ON }}\left(d_{1}\right)=Z$ si J est du type mauvais temps,

$F_{1 \mathrm{~N}}\left(d_{1}\right)=Z$ si $\mathrm{J}$ est du type beau temps.

On se fixe ainsi a priori que la première séquence de la journée à compter du lever du soleil est un passage nuageux (compte tenu du fait que les dispositifs solaires sont le plus souvent à l'arrêt au lever du soleil, cette simplification ne paraît pas introduire de biais pratique; toutefois on pourrait également, si on le préfère, tirer au hasard si la première séquence est une éclaircie ou non).

La deuxième séquence, du type opposé à la première, sera simulée de la même façon avec un nouveau tirage $\operatorname{de} Z$ :

$$
F_{\mathrm{OE}}\left(d_{2}\right)=Z \quad \text { ou } \quad F_{1 \mathrm{E}}\left(d_{2}\right)=Z
$$

selon le type de temps de J.

On génère ainsi $n$ séquences en tronquant la dernière s'il le faut pour respecter la longueur du jour.

3.2.3 Contrôle de la simulation des longueurs des séquences. - Les figures 5 et 6 permettent de comparer les fonctions de répartition empiriques et simulées $\mathrm{du}$ mois de juillet. On constate une bonne concordance, vu l'effectif réduit de l'échantillon. 

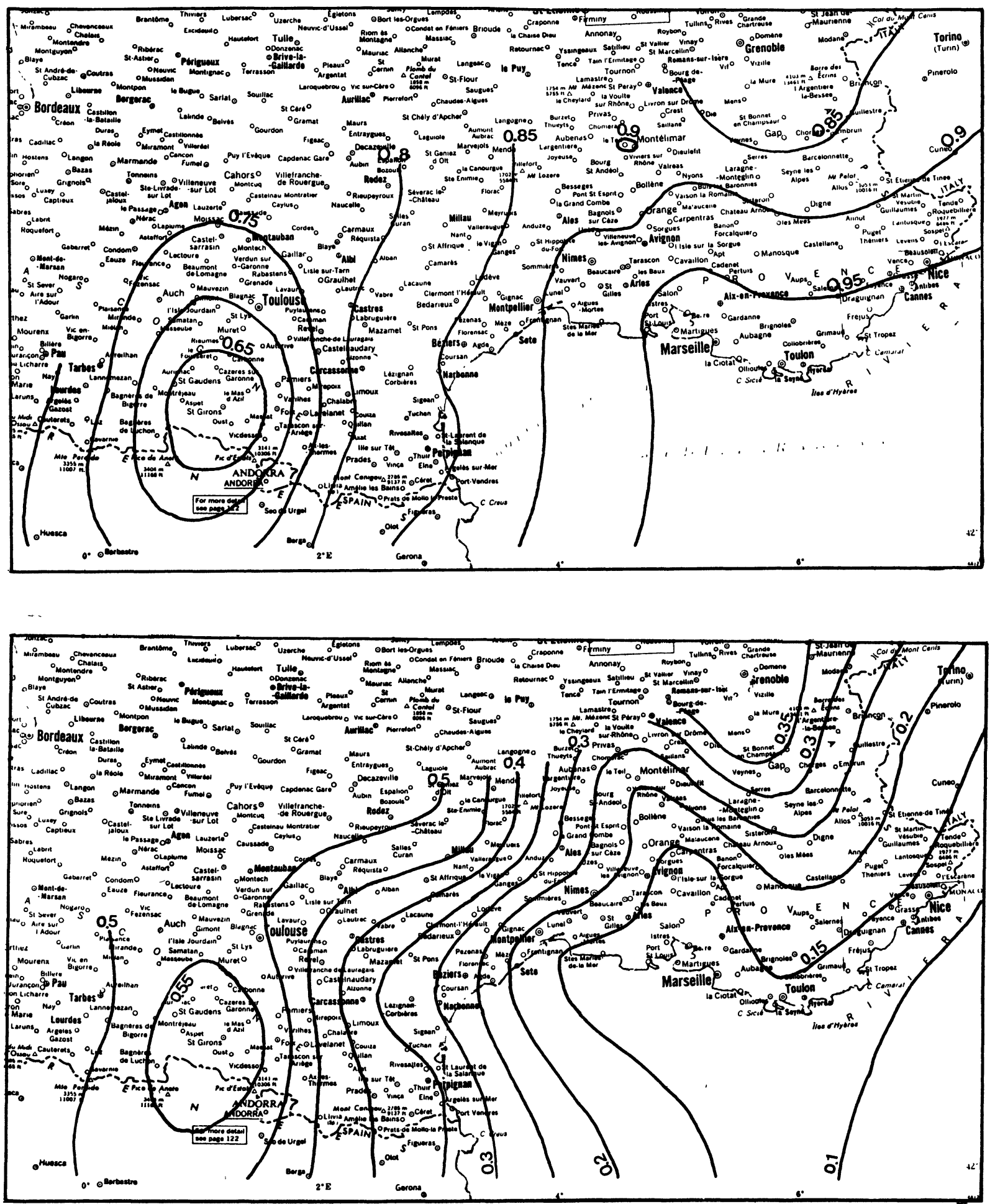

Fig. 2. - Courbes isovaleur des probabilités de passage beau temps-beau temps $P_{11}$ en haut; et mauvais temps-mauvais temps $P_{00}$ en bas, calculées sur l'ensemble des mois de juillet de 1949 à 1975.

[Isovaleur level contours for the probabilities $P_{11}$ (fine weather-fine weather, upper map) and $P_{00}$ (bad weather-bad weather, lower map) for the month of July in the southern part of France.]

Toutefois, il nous restait à vérifier que l'hypothèse d'indépendance des durées des séquences successives était justifiée, à savoir que la durée d'une éclaircie ne dépend pas de la durée du passage nuageux précédent et inversement. La figure 7 donne la durée observée $L_{0}$ d'un passage nuageux suivi d'une éclaircie de durée $L_{1}$; la figure 8 concerne la simulation. On ne constate pas de différences notables. 


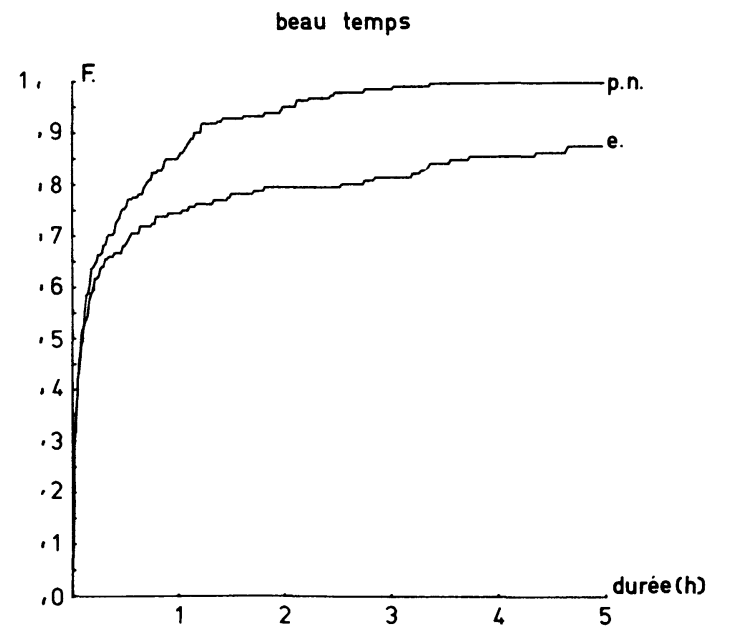

Fig. 3. - Fonction de répartition des durées des séquences de passages nuageux (p.n.) et d'éclaircies (e.) pour les jours de beau temps ì Saint-Chamas en juillet 1977.

[Repartition function of the duration of cloudiness (p.n.) and sunshinning (e.) for the fine weather days at Saint-Chamas in July.]

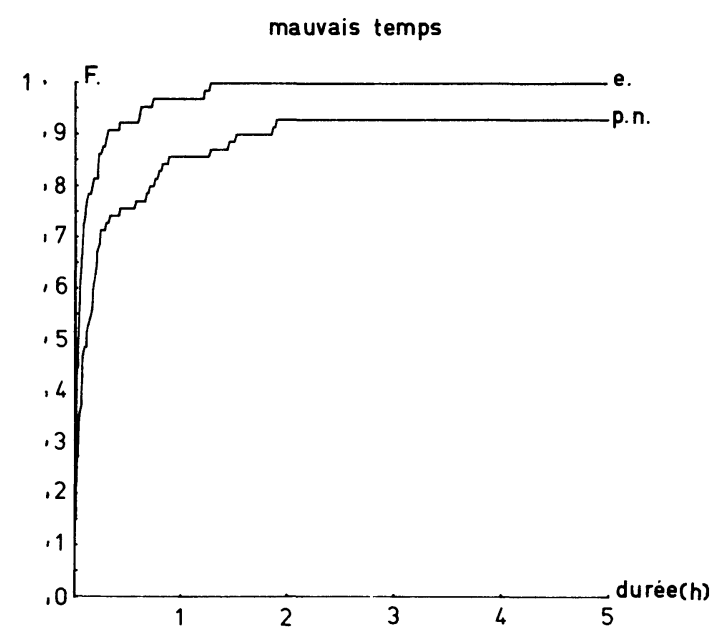

Fig. 4. - Fonction de répartition des durées des séquences de passages nuageux (p.n.) et d'éclaircies (e.) pour les jours de mauvais temps à Saint-Chamas en juillet 1977.

[Same as figure 3, but for bad weather days.]

3.3 AfFeCtation Des VALeURS DE L'ÉClairement DIRECT AU SEIN DES ÉClAIRCIES OU DES PASSAGES NUAGEUX. - L'affectation est très simple :

- Au sein d'un passage nuageux $I=0$.

- Au sein d'une éclaircie, on impose $I=I_{\max }(t)$, $I_{\max }(t)$ étant l'éclairement maximal à l'instant $t$ calculé de la façon suivante : sur les données du mois de juillet 1977 , on a ajusté, par moindres carrés, la fonction $a(\sin h(t))^{b}$, où $h(t)$ est la hauteur angulaire du soleil à l'instant $t$, aux maxima mesurés aux instants $t$ de l'ensemble des jours de juillet (en prenant, en fait, le maximum par intervalle de $15 \mathrm{mn}$ ). On a trouvé

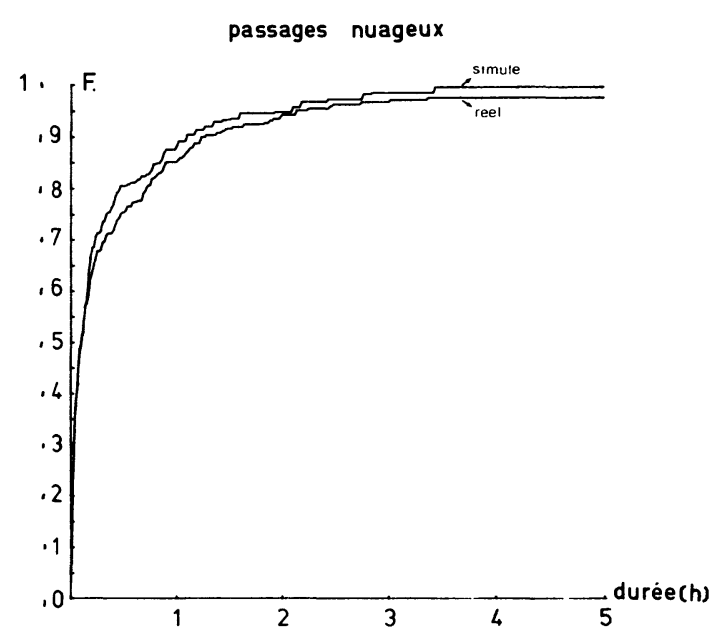

Fig. 5. - Fonctions de répartition à partir des données réelles (juillet 1977) et des données simulées des durées des passages nuageux (tous jours confondus).

[Repartition functions of the duration of cloudiness for real and simulated data (both types of weather added).]

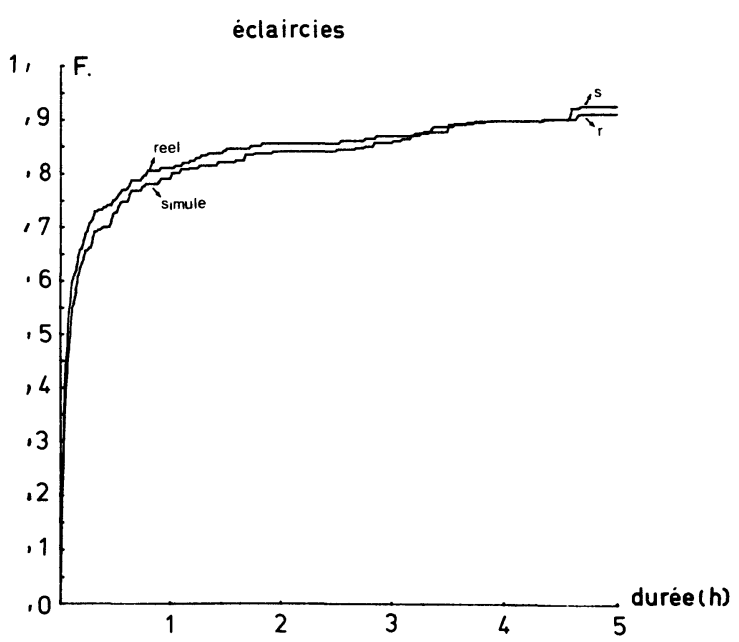

Fig. 6. - Fonctions de répartition à partir des données réelles (juillet 1977) et des données simulées des durées des éclaircies (tous jours confondus).

[Same as figure 5, but for sunshinning.]

$a=1036 \mathrm{~W} / \mathrm{m}^{2}, b=0,51$ et une corrélation de 0,94 entre les logarithmes des valeurs maximales observées et $\log (\sin h(t))$.

3.4 RÉSULTATS ET CONCLUSIONS DU SCHÉMA I. On a simulé de cette façon plusieurs échantillons de 31 jours. Aux comparaisons précédemment développées, nous ajouterons l'analyse des histogrammes de fréquence des valeurs obtenues chaque minute sur l'ensemble des jours. La figure 9 donne l'histogramme réel ainsi qu'un histogramme des données simulées. On constate des différences notables pour les valeurs 


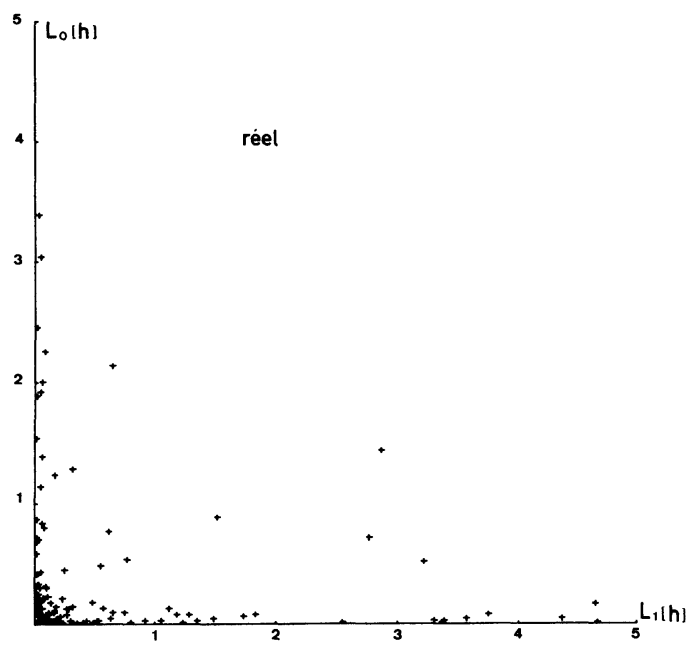

Fig. 7. - Durée $L_{1}$ d'une éclaircie précédée d'un passage nuageux de durée $L_{0}$ (données réelles, juillet 1977).

[Duration $L_{1}$ of sunshinning as a function of the duration of the preceeding cloudiness duration $L_{0}$ (real data).]

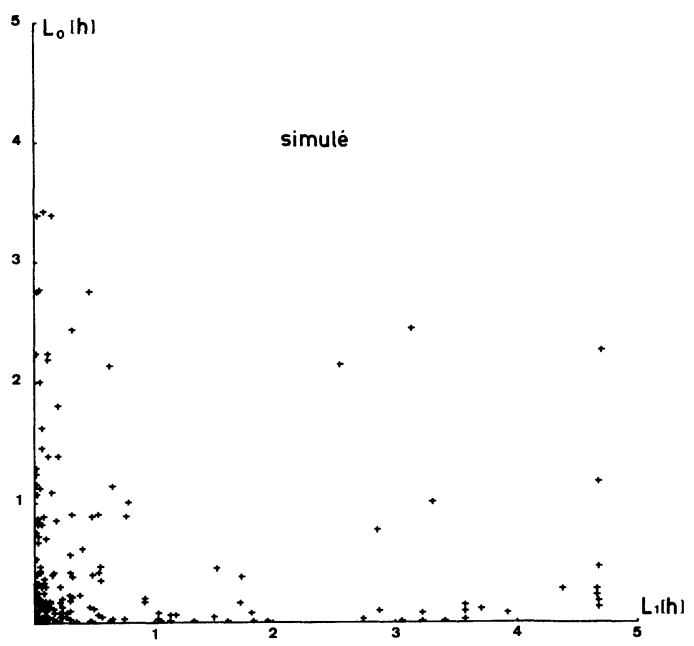

Fig. 8. - Durée $L_{1}$ d'une éclaircie précédée d'un passage nuageux de durée $L_{0}$ (données simulées).

[Same as figure 7, but for simulated data.]
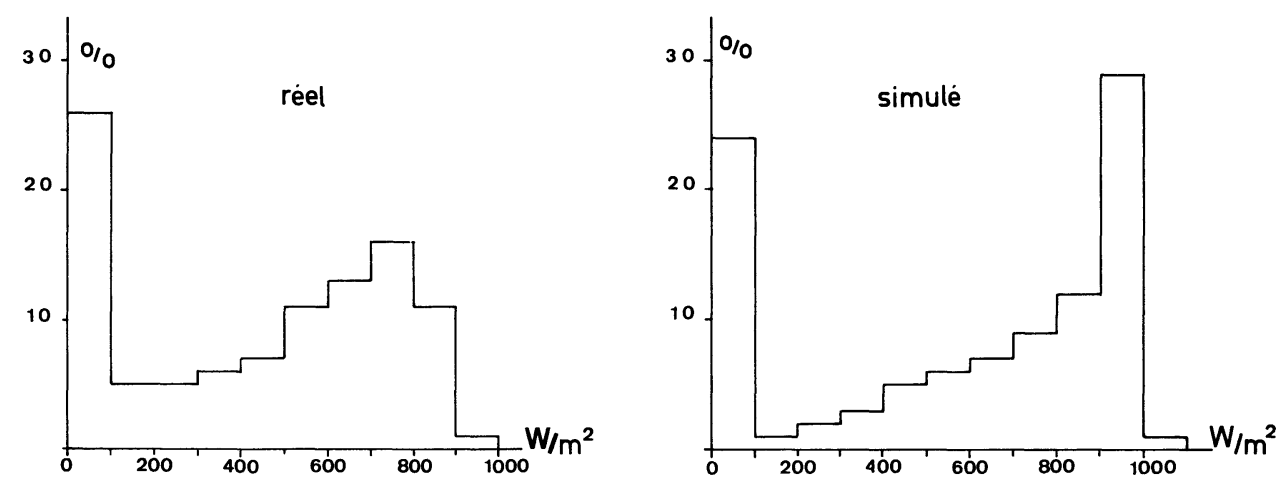

Fig. 9. - Histogramme des valeurs de l'éclairement direct à la minute, réelles (juillet 1977) et simulées selon le schéma $I$.

[Histogram of the instantaneous direct radiation (real data, and simulated data resulting of the application of scheme I).]

élevées de l'éclairement. Ce biais peut avoir deux causes :

- le schéma à 2 états journaliers est trop simpliste ;

- l'affectation $I=0$ ou $I=I_{\max }$ est mauvaise.

Ceci nous a conduit à modifier dans un premier temps, la génération du type de temps, donc à modifier la simulation des chroniques de jours.

4. Schéma II : modèle à trois états journaliers et à tout ou rien pour l'éclairement. - La seule différence par rapport au modèle précédent est que nous définissons trois types de journées au lieu de deux.

4.1 DéFINITION DE TROIS ÉTATS JOURNALIERS. $\mathrm{Au}$ vue des histogrammes journaliers de l'éclairement direct à la minute, nous avons défini trois états journaliers :

Etat $0:$ Jours totalement ou fortement couverts $\bar{I}$ journalier inférieur à $200 \mathrm{~W} / \mathrm{m}^{2}$ (I étant la moyenne journalière de l'éclairement direct reçu chaque minute du lever au coucher du soleil), ce qui statistiquement correspond à une insolation inférieure à $40 \%$ de l'insolation potentielle. L'histogramme des valeurs instantanées de l'éclairement direct a un mode important entre 0 et $100 \mathrm{~W} / \mathrm{m}^{2}$ et une forme de $L$.

\section{Etat 1 : Jours à passages nuageux}

Pour ces jours-là, l'histogramme des valeurs instantanées a deux modes : l'un entre 0 et $100 \mathrm{~W} / \mathrm{m}^{2}$, l'autre vers $700-800 \mathrm{~W} / \mathrm{m}^{2}$, soit une forme de $U$. Ces jours correspondent à :

$I$ journalier compris entre 200 et $600 \mathrm{~W} / \mathrm{m}^{2}$ soit à une fraction d'insolation $\mathrm{SS} / \mathrm{SS}_{0}$ entre 0,4 et 0,9 .

\section{Etat 2 : Jours dégagés}

L'histogramme des valeurs instantanées n'a plus qu'un mode vers $800-900 \mathrm{~W} / \mathrm{m}^{2}$, d'où une forme en $\mathrm{J}$. Ces jours correspondent à :

$\bar{I}$ journalier supérieur à $600 \mathrm{~W} / \mathrm{m}^{2}$ soit une fraction d'insolation supérieure à 0,9 . 
4.2 Simulation en Chaine de Markov des types DE TEMPS JOURNALIERS. - A partir des longues séries d'insolation à Marseille-Marignane, nous avons déterminé les probabilités marginales des 3 états ainsi que la matrice de passage d'un état journalier à l'état du lendemain :

$$
\begin{aligned}
& P_{0}=0,039 \text { Probabilité } \frac{\mathrm{SS}}{\mathrm{SS}_{0}} \text { inférieur à } 0,4 \\
& P_{1}=0,691 \quad \text { Probabilité } \frac{\mathrm{SS}}{\mathrm{SS}_{0}} \text { entre } 0,4 \text { et } 0,9 \\
& P_{2}=0,270 \quad \text { Probabilité } \frac{\mathrm{SS}}{\mathrm{SS}_{0}} \text { supérieur à } 0,9 .
\end{aligned}
$$

La matrice de passage est la suivante :

\begin{tabular}{lcccc} 
& & \multicolumn{3}{c}{ Etat du jour J +1} \\
Etat du Jour J & & 0 & 1 & 2 \\
& & - & - & - \\
Mauvais & 0 & 0,065 & 0,806 & 0,129 \\
Moyen & 1 & 0,044 & 0,770 & 0,185 \\
Beau & 2 & 0,023 & 0,465 & 0,512
\end{tabular}

Ainsi pour un jour quelconque $\mathrm{J}$, il y a une probabilité de $0,691=P_{1}$ que ce soit un jour à passages nuageux (Etat 1), et si ce jour est effectivement à passages nuageux, il y a une probabilité $P_{12}=0,185$ que le jour $\mathbf{J}+1$ soit dégagé (Etat 2).

4.3 Simulation et Résultats. - 4.3.1 Simulation de la chronique des états journaliers. - Premier jour : On tire au hasard $Z$ uniforme entre 0 et 1 :

si $Z \leqslant P_{0}$ le $1^{\text {er }}$ jour est mauvais (Etat 0 ),

si $P_{0}<Z \leqslant P_{1}$ le $1^{\mathrm{er}}$ jour est moyen (Etat 1 ),

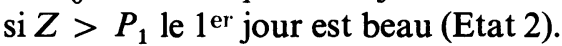

\section{Jours suivants :}

Soit $k$ ( $k=0$ ou 1 ou 2) l'état d'un jour, pour déterminer l'état du lendemain on gènère $Z$ uniforme sur 0 et 1 :

si $Z \leqslant P_{k 0}$ le lendemain est mauvais (Etat 0 ), si $Z$ est compris entre $P_{k 0}$ et $P_{k 1}$ le lendemain correspond à l'Etat 1 ,

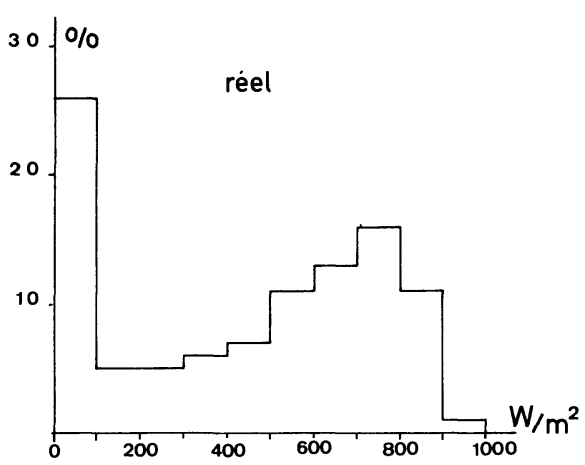

si $Z$ est supérieur à $P_{k 1}$ le lendemain est beau (Etat 2).

Cette génération respecte donc les probabilités marginales ainsi que les probabilités conditionnelles à 1 jour.

4.3.2 Simulation au sein des jours. - Elle est identique dans son principe à celle du schéma $\mathrm{I}$, sinon que les fonctions de répartition des durées des séquences des éclaircies ou des passages nuageux sont définies pour trois types de temps, d'où six fonctions de répartition au lieu de quatre. L'affectation des valeurs au sein des éclaircies ou des passages nuageux reste la même : $I=0$ ou $I=I_{\max }$ fonction de la date et de l'instant considéré.

4.3.3 Résultats et conclusions. - La figure 10 donne les histogrammes de fréquences des valeurs à la minute. On constate que l'amélioration entre le schéma II et le schéma I est faible : il y a encore trop de fortes valeurs.

Nous en avons conclu qu'il fallait chercher une amélioration dans l'affectation des valeurs de l'éclairement au sein des séquences de passages nuageux ou d'éclaircies et que l'on pouvait, par souci de simplification, revenir à deux états journaliers.

5. Modèle à deux états journaliers et à éclairement simulé d'après les données réelles. - Ce modèle est identique au premier en ce qui concerne : liers ;

- la génération des chroniques des états journa-

- la génération des longueurs de séquences de passages nuageux ou d'éclaircies selon l'état journalier.

Aussi, ne reprendrons-nous pas les explications déjà fournies.

5.1 Simulation des Valeurs De l'éClairement AU SEIN DES ÉCLAIRCIES OU DES PASSAGES NUAGEUX SEION LE TYPE DE TEMPS ET LE MOMENT CONSIDÉRÉ. 5.1 .1 Nécessité de distinguer les types de temps journaliers. - On constate que les valeurs de l'éclairement que ce soit au sein des éclaircies ou au sein des

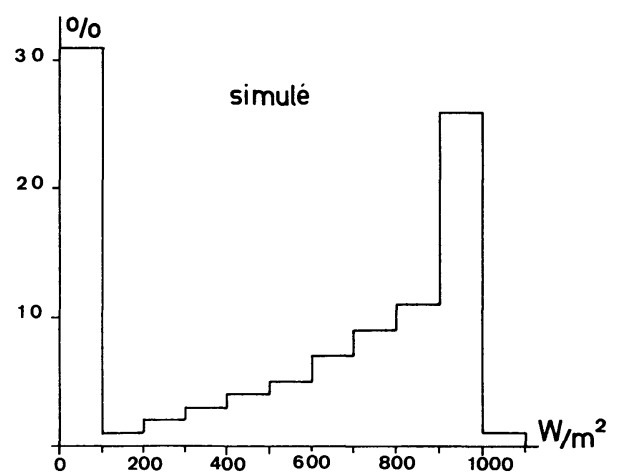

Fig. 10. - Histogramme des valeurs de l'éclairement direct à la minute, réelles (juillet 1977) et simulées selon le schéma II.

[Same as figure 9, but for scheme II.] 
passages nuageux sont assez différentes selon qu'il s'agit d'un mauvais jour (Etat 0 ) ou d'un beau jour (Etat 1). Aussi avons-nous par la suite, étudié séparément ces deux types de temps.

5.1.2 Nécessité de tenir compte de l'heure de la journée. - On constate également qu'au sein d'un même type de temps, la fonction de répartition des éclairements n'est pas la même le matin qu'à midi ou le soir pour deux raisons :

- astronomique d'une part (hauteur du soleil différente);

- météorologique (exemple orages du soir plus fréquents que le matin, en été).

Aussi, avons-nous déterminé les fonctions de répartition des valeurs instantanées du rapport $I / I_{\max }(h)$ au sein des éclaircies et des passages nuageux en fonction :

- du type de temps,

- de l'heure.

La figure 11 présente l'histogramme empirique des rapports $I / I_{\max }$ de 10 à $11 \mathrm{~h}$ et de 15 à $16 \mathrm{~h}$ pour les éclaircies et les passages nuageux. On notera que ces histogrammes, certes définis sur des petits échantillons sont assez différents les uns des autres.

5.2 Applications. - Nous avons d'abord simulé ainsi les données à la minute : au sein d'une séquence, et pour chaque minute, il faut tirer un nombre $Z$

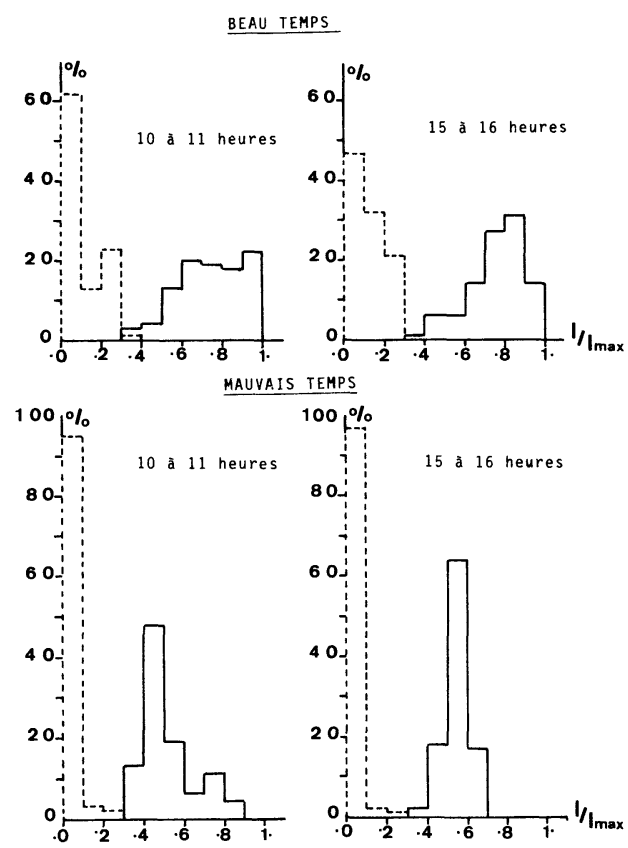

Fig. 11. - Histogramme de fréquences des fractions $I(t) / I_{\max }(t)$ de 10 à $11 \mathrm{~h}$ et de 15 à $16 \mathrm{~h}$ du mois de juillet 1977 , pour les éclaircies (trait plein) et les passages nuageux (tirets). $I(t)$ étant l'éclairement direct observé à l'instant $t$ et $I_{\max }(t)$ l'éclairement maximal à l'instant $t$ défini dans le $\$ 3.3$.

[Histogram of the ratios $I / I_{\max }$ observed between 10 and 11 a.m and between 3 and 4 p.m. during sunshinning periods (continuous lines) and cloudiness periods (broken lines).] puis lire dans un tableau, la valeur de $I / I_{\max }$ déduite de la fonction de répartition correspondante au type de temps, au type de séquence et à l'heure de la journée. Le calcul est très long, aussi avons-nous simplifié en considérant qu'au sein d'une séquence, on ne changeait la valeur de $I / I_{\max }$ que toutes les dix minutes, si la séquence dure plus de dix minutes; $I$ varie alors au sein de ces dix minutes comme $I_{\max }$, qui est fonction $\mathrm{du}$ temps solaire. On pourrait d'ailleurs ajouter à cette valeur un bruit pour simuler les faibles fluctuations.

Une telle simulation suppose donc connues :

- les probabilités marginales et conditionnelles des 2 états journaliers; soit 2 valeurs à définir $P_{01}$ et $P_{10}$;

- les fonctions de répartition des durées des séquences des passages nuageux ou éclaircies selon le type de temps soit 4 fonctions ;

- les fonctions de répartition de $I / I_{\max }$ selon le type de temps, le type de séquences et l'heure (nous avons utilisé 4 fonctions par heure, on pourrait sans doute regrouper 2 ou $3 \mathrm{~h}$ ) soit de 20 à 60 fonctions.

Une telle simulation peut se faire à partir d'un ordinateur de capacité moyenne; sur un ordinateur du type CII Iris 80 il faut une douzaine de minutes pour simuler ainsi un mois de données.

5.3 RÉSUlTATS ET COMPARAISON AVEC LA RÉALITÉ. - Les résultats des chroniques de jours et de longueurs de séquences sont les mêmes qu'avec le premier schéma; il nous reste à vérifier si la génération des valeurs de l'éclairement au sein des séquences est meilleure. La figure 12 présente les histogrammes des valeurs instantanées réelles et simulées sur 31 jours de caractéristiques assez voisines à la série historique de calage. La différence s'explique sans doute en partie par la petitesse de l'échantillon, mais l'amélioration est très nette par rapport aux schémas I et II.

Les figures 13 et 14 donnent des exemples de journées réelle et simulée, de moyennes voisines. La simulation paraît très réaliste.

6. Conclusions et perspectives. - 6.1 COMPARAISON DES TROIS MODÈLES. - Les deux premiers modèles fort simples présentent un biais très gênant : trop de valeurs fortes. Le troisième modèle est beaucoup plus satisfaisant. Rappelons qu'il respecte :

- les séquences de jours successifs et leur dépendance à 1 jour ;

- les longueurs des séquences nuages-éclaircies - selon le type de temps;

- les valeurs des éclairements au sein des séquences selon le type de temps, le type de séquence et le moment de la journée.

Les limites de ce modèle sont claires :

- L'autocorrélation de la variable aléatoire type de temps pour des jours séparés par un intervalle de 

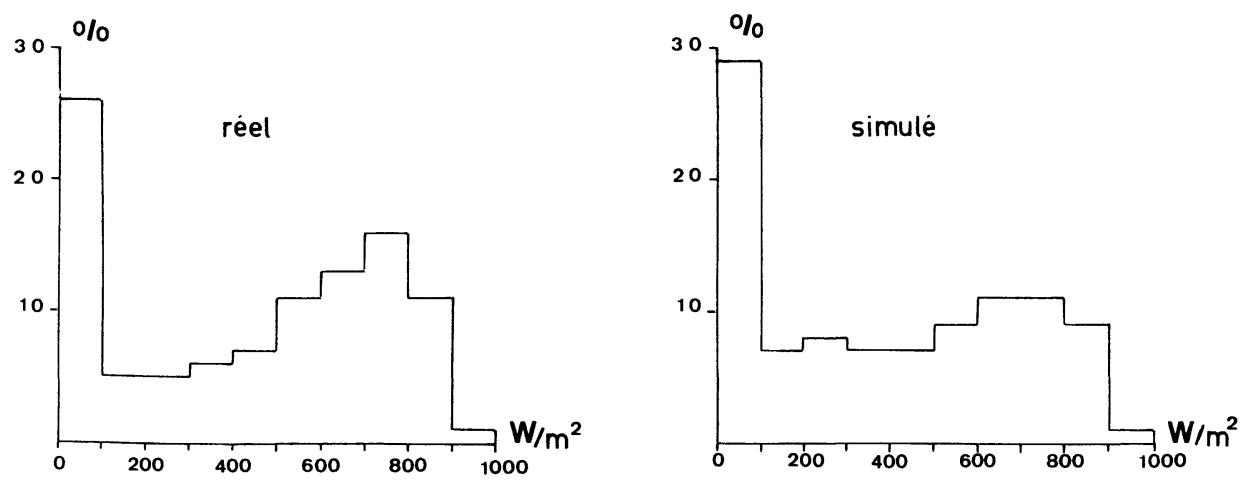

Fig. 12. - Histogramme des valeurs de l'éclairement direct à la minute, réelles (juillet 1977) et simulées selon le schéma III.

[Same as figure 9, but for scheme III.]

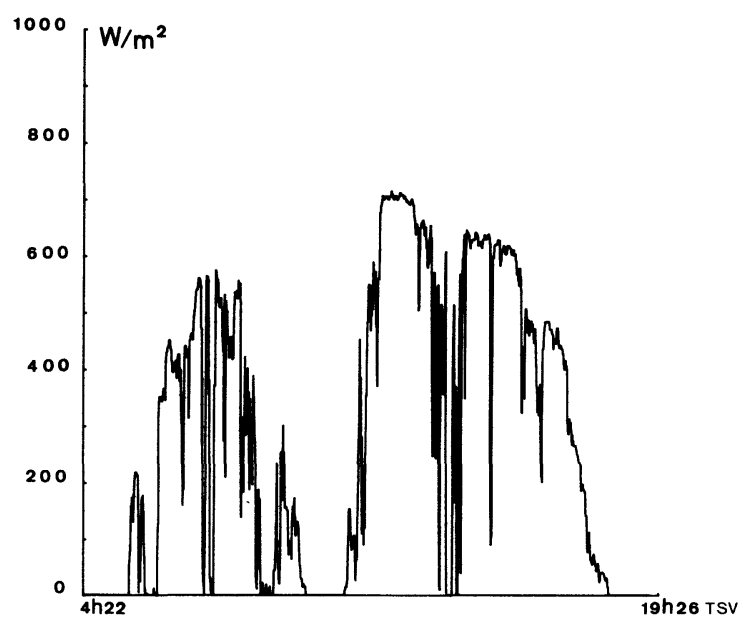

Fig. 13. - Exemple de journée réelle.

[Example of direct radiation recorded (real day).]

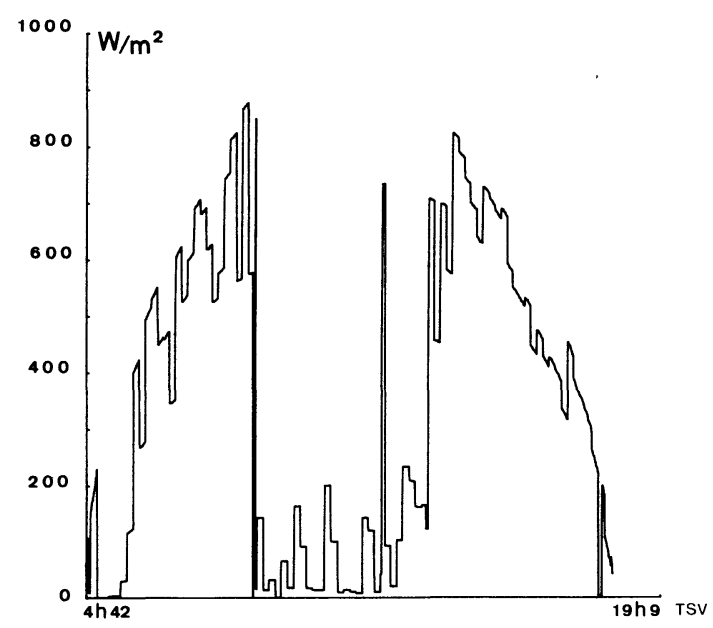

Fig. 14. - Exemple de journée simulée.

[Example of direct radiation record as simulated.] $n$ jours a la forme exponentiellement décroissante avec $n$ caractéristique des chaînes de Markov du premier ordre. On pourrait sans problème majeur, introduire les chaînes de Markov d'ordre plus élevé pour mieux rendre compte des corrélations à plus d'un jour, mais cela n'améliore pas de façon sensible la reproduction des séquences les plus longues d'un même type de temps (cf. J. Goussebaile [2]).

- Nous n'avons pas introduit l'autocorrélation des valeurs de l'éclairement au sein des éclaircies ou des passages nuageux, ce qui ne biaise pas les répartitions marginales mais conduit à des fluctuations trop rapides pour des tirages à faible pas de temps (la minute par exemple). L'utilisation du modèle pour étudier la gestion d'un système solaire à faible inertie exigerait d'introduire cette autocorrélation.

En outre, nous avons fait l'hypothèse que les durées des séquences étaient indépendantes des durées des séquences précédentes, hypothèse incertaine mais non contredite par l'expérience.

6.2 INTÉRÊT. - Si l'on veut tester un schéma d'utilisation de l'énergie directe dans un appareillage à faible inertie, on peut évidemment commencer par utiliser les séries historiques; ceci suppose la lecture de fichiers importants et est limité par la rareté actuelle de tels fichiers.

Par contre, on peut simuler de longues séries, ce qui élimine le problème de lecture de fichiers. Ces séries présentent un avantage parfois fondamental de simuler de façon réaliste un grand nombre d'éventualités : par exemple, on pourra examiner le fonctionnement de l'appareil lors de séquences de mauvais temps plus longues que celles observées mais de durée statistiquement probable, de même pour le beau temps...

Il reste d'une part à examiner dans quelle mesure on peut améliorer le modèle de simulation sans le rendre trop complexe, et d'autre part, à utiliser les données actuellement recueillies pour mieux définir toutes les lois de probabilité expérimentales utilisées. 
Remerciements. - Nous tenons à remercier la Météorologie Nationale et Electricité de France, notamment MM. Sicart et Gentili. Ce travail a été effectué dans le cadre de travaux du Programme Interdisciplinaire de Recherches et Développement de l'Energie Solaire (PIRDES) du CNRS.

\section{Bibliographie}

[1] Bois, Ph., Mejon, M. J., Analyse du gain d'information en fonction du pas de temps de mesure du rayonnement direct. Exemple de Saint-Chamas à l'échelle de la minute. Publication du CNRS : Analyse statistique des processus météorologiques appliquée à l'énergie solaire. Paris 1979, p. 145 à 158.

[2] Goussebaile, J., Analyses statistiques de données journalières d'insolation et d'irradiation. Applications au Sud-Est de la France. Thèse de Docteur-Ingénieur. Grenoble, septembre 1978.

[3] Lestienne, R., Obled, Ch., Bois, Ph. - Analyse temporelle et cartographie de la matrice stochastique pour le modèle Markovien dans le midi de la France. Publication du CNRS identique à [1] p. 353 à 380 .
[4] Lestienne, R., Modèle Markovien simplifié de météorologie à deux états. L'exemple d'Odeillo. La Météorologic 12 (1978).

[5] Mejon, M. J., Goussebaile, J., Bois, Ph. et Vachaud, G., Etude des liaisons entre l'irradiation solaire journalière et quelques variables météorologiques. Publication du CNRS identique à [1] p. 105 à 143.

[6] Perrin de Brichambaut, M., Cah. AFEDES no 1 (1975).

[7] Sicart, F., Gentili, B., Exploitation des mesures météorologiques dans le cadre des centrales solaires à tour. Publication du CNRS identique à [1] p. 185 à 207.

[8] Bourges, B., Corrélation entre l'irradiation solaire globale et la durée de l'insolation au niveau horaire. Centre d'Energétique (Ecole des Mines de Paris) mars, 1978. 